\title{
Training Experience in the US School Psychology Program: Understanding Asian International Students' Assets, Challenges, and Coping
}

\author{
Chunyan Yang ${ }^{1}$ (I) $\cdot$ Chun Chen $^{2} \cdot$ Meiki Chan ${ }^{2} \cdot$ Cixin Wang ${ }^{3} \cdot$ Hua Luo ${ }^{1} \cdot$ Xueqin Lin ${ }^{1}$
}

Accepted: 31 August 2020 / Published online: 10 September 2020

(C) California Association of School Psychologists 2020

\begin{abstract}
To address the rapidly increasing demand for culturally and linguistically diverse school psychologists and training needs for international school psychology students in the USA, this study was grounded in the risk and resilience framework and used semi-structured interviews to explore the nuanced experiences of Asian international trainees in school psychology programs in the USA. Participants included 11 Asian international students who were enrolled in or recently graduated from school psychology programs in the USA. Thematic analysis revealed that Asian international school psychology students identified some assets they have brought to the program, including their diverse perspectives and experiences, awareness and commitment to social justice, and the potential to diversify the school psychology workforce. Participants also identified several challenges they were facing, such as acculturative stress, microaggression and discrimination, training program-related issues, and working restrictions related to their visa status. Moreover, they identified some effective strategies (i.e., seeking resources and increasing internal strengths) they have used to cope with these challenges. The findings provide important implications for school psychology graduate programs in the USA to improve the training experiences and training outcomes for international students from Asia and other countries.
\end{abstract}

Keywords International students · Graduate student · Diversity $\cdot$ School psychology training

As ethnic minority students reached $50.5 \%$ of the student population in public schools in the USA in 2014 (Goldenberg 2014), there is an increasing demand for culturally and linguistically diverse school psychologists (National Association of School Psychologists 2017; Walcott et al. 2016). Many school psychology graduate programs in the USA have shown great interest in recruiting and retaining/ training graduate students to diversify the school psychology workforce (Clark et al. 2012). Although the exact number of international graduate students who are enrolled in the US

Chunyan Yang

yangcy@berkeley.edu

1 Graduate School of Education, University of California, Berkeley, 2121 Berkeley Way, Berkeley, CA 94720 , Berkeley, CA 94720-1670, USA

2 Department of Counseling, Clinical, and School Psychology, University of California, Santa Barbara, USA

3 Department of Counseling, Higher Education, and Special Education, University of Maryland, College Park, USA school psychology programs remains unknown, anecdotal information suggests that the number of international students from Asia has rapidly grown in the US school psychology programs. According to the Association of Chinese Helping Professionals and Psychologists-International (ACHPPI), over the past 10 years, there have been more than 150 Chinese international students who have come to the USA and are receiving or have completed graduate-level training in school psychology. Although Asian international students make up a significant portion of overall international students enrolled in the program, an empirical understanding of Asian international graduate students' training experiences in the school psychology programs in the USA is lacking. Such an understanding is critical to support the training and career preparation of international students in school psychology in the USA. To fill this gap, the present study used semi-structured interviews to explore Asian international trainees' perceptions of their assets, challenges, and strategies during their training in the school psychology program in the USA. The findings would provide implications for US school psychology graduate programs to promote 
positive training experiences and international students' training outcomes.

\section{Assets of International Students}

According to a report from the Institute of International Education (2019), there were over 37,000 international students enrolled in graduate-level programs in the USA during the 2018-2019 academic year. With the increasing number of international students in the US universities, the contribution of international students' role in enriching the diversity of campus culture and students' intellectual life has been recognized by many researchers (Choudaha and Chang 2012; Grayson 2008). Faculty members also identify the benefits of international graduate students, including filling research assistant vacancies, helping faculty members establish international ties, and providing different perceptions for domestic students (Trice 2003). However, there is no scientific understanding of how international graduate students perceive their assets and contributions. Moreover, the majority of existing studies have focused on the experiences of international graduate students in the STEM field. Much less research has studied their experiences in the field of social science. To date, no empirical study has been conducted to understand the experiences of international graduate students in school psychology programs in the USA.

\section{Challenges Faced by International Students}

When international students leave their home countries and come to the USA to seek a high-quality education, they face many challenges, including language barriers, heavy financial burden, lack and loss of social support, limited abilities to manage the family crisis from a distance (Wedding et al. 2009), and difficulties in navigating the complexity of cultural norms and sociopolitical histories (Lee 2013; Park-Saltzman et al. 2012). International students may also experience feelings of isolation, microaggression, discrimination by their peers and professors because of cultural differences, and demographic mismatch (Reid and Dixon 2012). Also, the visa application and the search for work permit sponsorship can be challenging for international students when they are seeking their predoctoral and postdoctoral training in the USA (Lee 2013). Visa and language barriers limit international students' opportunities for employment and professional resources (Wedding et al. 2009). In the literature, there are a few studies that identify the challenges that are unique to psychology students who are enrolled in programs with clinical training components. For example, Wedding et al. (2009) summarized that international psychology students might experience cultural values that conflict with their clients (e.g., parenting, sex, alcohol, and drugs), and find it difficult to empathize and understand their clients' problems (Wedding et al. 2009). However, these studies were based on students' experiences from counseling and clinical psychology programs; none of them have focused on the experiences of students in the school psychology graduate programs in the USA.

\section{Coping Strategies Used by International Students}

To address the challenges faced by international students, it is important to understand the strategies they have used and found to be effective in coping with these challenges during their training. To date, no research study has been conducted to identify such strategies. However, some existing research focusing on the broader groups of international students has provided some helpful insights. For example, international students were found to use their internal strengths to cope with their challenges, such as being confident about their differences, standing up for themselves, and persevering (Mittal and Wieling 2006). Other types of coping strategies (i.e., task-oriented, emotion-oriented, and avoidance-oriented) were also used by international students to reduce acculturative stress (Ra and Trusty 2015). Moreover, resilience factors, such as being positive (world and yourself), focused, flexible (social and thoughts), organized, and proactive, were also identified as positive coping strategies for international students (Wang 2009). In addition to relying on coping strategies, seeking external support and resources, such as mentoring relationships, religious belief, and campus services, has been identified as another way for international students to manage their difficulties (Park et al. 2017). While some effective strategies have been identified, most studies have focused on the general population of international students. So far, no study has examined what strategies Asian international students in the school psychology program use to cope with the challenges they face during their academic and clinical training.

\section{Risk and Resilience Framework}

The guiding research questions of the present study were conceptualized based on the risk and resilience framework. The risk and resilience framework describes the process in which individuals overcome risks and avoid adverse outcomes by using internal assets and external resources when confronted with stressful situations (Fergus and Zimmerman 2005). In the context of international students' training experiences with the school psychology program, it is important to not only understand the challenges or risk factors faced by international school psychology students, but also use a resilience 
framework to identify the assets or protective factors in their training experiences to develop effective strategies that could promote Asian international students' resilience and positive training outcomes. To date, existing studies about international students' training experiences have primarily focused on the risk factors, and more empirical studies are needed to advance our understanding of their experience from both risk and resilience perspectives.

\section{Present Study}

Guided by the risk and resilience framework, the current study explored Asian international students' experiences during their training in the US school psychology programs. We conducted semi-structured interviews with eleven Asian international students and used thematic analysis to explore three research questions: (1) What are the main assets perceived by Asian international students during their training in the school psychology program? (2) What are the challenges they were facing during their school psychology training? (3) What are some effective strategies they used to cope with these challenges?

\section{Method}

A qualitative research methodology was adopted because the topic under investigation was in its exploratory phase. Based on the direct responses from the research participants, the use of this methodology and individual interviews also allowed for a deep understanding of Asian international school psychology trainees' experience in the USA.

\section{Participants}

Nine participants $(82 \%)$ were female, and two (18\%) were male. Their ages ranged between 23 and 42 years, with a mean age of 29.5 years. The countries of origin were China $(n=6$ in Mainland China and $n=3$ in Hong Kong), Japan $(n=1)$, and India $(n=1)$. Two participants (one from Hong Kong and one from India) reported English as their native language. Participants have lived in the USA from a range of 3 to 18.5 years, with a mean of 5.2 years. Participants included seven Asian international graduate students who were enrolled in US school psychology programs and four individuals who recently graduated from a US school psychology program at the time of data collection. Three of the participants graduated from or were pursuing an Education Specialist (i.e., Ed.S.) degree in school psychology. Eight of them graduated from or were pursuing a doctoral degree in APA-accredited school psychology programs. All of them were living in the USA at the time of data collection. Four participants obtained their undergraduate and master's degrees in the USA, and the other seven students completed their prior education in Asia before joining the US school psychology programs.

\section{Research Team}

Both the first and fourth authors are Asian faculty members in APA-accredited school psychology programs in the USA, and they both came to the USA as international students and obtained graduate training in school psychology. The second and third authors are currently enrolled as 3rd- and 2nd-year doctoral students in a school psychology doctoral program. The fifth and sixth authors are incoming doctoral students in another doctoral school psychology program. All research team members are fluent in both English and Chinese. Five of the six researchers are native speakers in Mandarin Chinese, and the other researcher is a native speaker in Cantonese.

\section{Data Collection Procedure}

Participants were recruited using a convenient and snowball sampling method. Invitations were made via personal contacts, social media, and e-mails sent out by research team members. Participation was on a voluntary basis. Written consent was obtained prior to participation. No monetary incentive was provided for participation. The study was approved by the Human Subjective Review Board of the researchers' academic institution.

Data were collected through an online demographic survey and videoconferencing via a Zoom platform. The demographic questionnaire included questions about gender, age, nationality, and educational backgrounds. The interviews were semi-structured and lasted approximately 30 to $40 \mathrm{~min}$. Each interview started with a broad question: "How do you feel about the school psychology training you have received?" To ensure consistent information across participants in the study, interviewees were asked to (1) describe their overall experience in the school psychology programs; (2) describe their assets and contributions to the school psychology program across personal, interpersonal, and environmental aspects; (3) describe the personal, interpersonal, and environmental challenges they are facing as a trainee; and (4) describe the coping strategies/resources they feel useful in the face of those challenges. Probing questions were used for clarification or elaboration of interviewees' responses. Respondents were interviewed in their preferred language ( $n=6$ in Mandarin, $n=3$ in Cantonese, $n=2$ in English). Interviews were transcribed into Mandarin or English verbatim. The coding process was conducted using the original language of the interview. The Chinese quotes were translated into English by the bilingual researcher team when the manuscript was prepared. 


\section{Data Analysis}

A descriptive thematic analysis procedure was used for analyzing the interview data (Braun and Clarke 2006). The thematic analysis included two stages. In stage one, the first author read all the transcripts and developed the overarching coding rules for the three established coding frames: (1) assets, (2) challenges, and (3) coping. A meeting led by the first author was held to train three graduate research assistants about the coding process. To develop the codebook, the team also coded one transcript to establish the code labels and definitions. Specifically, each text segment was first designated as a challenge, an asset, or a strategy. Text segments reflecting each of the three coding frames were highlighted in different colors. Each text segment was assigned with a code label, capturing the general meaning of the text segment. In stage two, two coders independently coded a transcript using the codebook. The initial intercoder reliability was $25 \%$ using the formula proposed by Campbell et al. (2013; Percentage of agreement $=$ Number of matched lines $/$ Total number of lines coded). Afterward, the coders met with the first author and other graduate research assistants to discuss any disagreements and clarify the coding rules using a negotiated agreement approach (Campbell et al. 2013). Then, these two researchers coded another transcript independently and reached the final intercoder reliability of $65 \%$. Because the intercoder reliability reached an adequate level (Campbell et al. 2013), these two coders used the same coding rules to code the rest of the transcripts. After the coding process was completed, the code labels were then grouped to generate subthemes. As an example, the following text segment, as "we would like them to acknowledge us that we bring our world view to [the] table" and "as assets, I would say I've had experiences in clinical psychology, and I've done a few work in community psychology. I see the inter-relationship between [the subjects]," would be coded under the subtheme "Diverse Perspectives and Experiences" in Assets.

\section{Results}

The purpose of the study was to explore the overall experiences of Asian international students in school psychology programs in the USA. Participants' responses reflected three general themes, including (1) assets, (2) challenges, and (3) coping. Table 1 presents the frequencies of the themes and subthemes reflected in the text segments of the interviewee's transcripts and the percentages of participants reporting the themes and subthemes. Figure 1 illustrates the visual presentations of the themes and subthemes examined in the study. Participants were assigned pseudonyms to maintain anonymity.

\section{Theme 1: Assets}

Most participants $(90.9 \%, n=10)$ noted that they have brought some assets into their school psychology programs and the field of school psychology. These assets can be categorized into three subthemes: (1) diverse perspectives and experience, (2) awareness and commitment to social justice, and (3) potential to diversifying the school psychology workforce.

\section{Diverse Perspectives and Experiences}

Most participants (63.6\%) identified having different cultural perspectives as one of the main assets they brought into their programs. Some participants referred the different cultural attitudes to their unique cultural beliefs and values, their points of view based on the "history and values of their country of origin," and their own "experiences as international students" in the USA. Some participants also reported that they started to become aware of their unique asset when seeing how other students and faculty expressed keen interest in learning from their cultural perspectives. For example, one participant explained:

I think that one advantage is that we did not grow up in this [U.S.] system, so I think sometimes our perspectives are new [to my classmates who grew up in the U.S.]. For example, in class, they might talk about global education or global healthcare. At least you have lived in another system, so you can provide new opinions. ... I think this might be a strength, and Americans like that. I think they [American peers in the school psychology program] are quite open to some different ideas. (Vanessa, a Hong Kong international student).

Apart from the unique international backgrounds of the participants, one participant recognized that her previous working and educational experiences and her personal life experiences (e.g., being a parent) helped her bring more new perspectives into school psychology. Particularly, the participant noted that "as assets, I would say [that] I've had experiences in clinical psychology, and I've done a few work in community psychology. Now when I finally come to school psychology, I see the inter-relationship between [them]" (Mary, an Indian international student).

\section{Potential to Diversify School Psychology Workforce}

About $45.5 \%$ of participants noted their potential to diversify the school psychology workforce by serving the increasingly diversified students and families in school psychology. They stated that their multicultural and multilinguistic knowledge and skills could better prepare them to become practitioners 
Table 1 Themes and subthemes related to experiences of school psychology Asian international students

\begin{tabular}{|c|c|c|c|}
\hline Themes and subtheme & Frequency reported & $N(11)$ & $\%$ of $N$ \\
\hline Assets & 30 & 10 & 90.9 \\
\hline - Diverse perspectives and experiences & 18 & 7 & 63.6 \\
\hline - Potential to diversifying school psychology workforce & 9 & 5 & 45.5 \\
\hline - Awareness and commitment to social justice & 3 & 3 & 27.3 \\
\hline Challenges & 131 & 11 & 100 \\
\hline - Acculturative stress & 24 & 11 & 100 \\
\hline - Microaggression and discrimination & 34 & 11 & 100 \\
\hline - Training program-related issues & 62 & 11 & 100 \\
\hline - Program's lack of experience working with international students & 45 & 11 & 100 \\
\hline - Representation of minority status & 17 & 8 & 72.7 \\
\hline • Funding & 11 & 6 & 54.5 \\
\hline - Job restriction related to visa status & 11 & 5 & 45.5 \\
\hline Coping & 67 & 9 & 81.8 \\
\hline - Seeking for resources & 50 & 9 & 81.8 \\
\hline - Seeking for social support & 22 & 8 & 72.7 \\
\hline - Seeking for professional resources & 19 & 7 & 63.6 \\
\hline - Finding role models & 5 & 5 & 45.5 \\
\hline - Seeking for demographic match & 4 & 2 & 18.2 \\
\hline - Increasing internal strengths & 17 & 7 & 63.6 \\
\hline - Increasing social-emotional strengths & 15 & 7 & 63.6 \\
\hline - Setting goals to improve motivation & 2 & 2 & 18.2 \\
\hline
\end{tabular}

Percentages indicate the percent of participants who mentioned each theme/subtheme at least once during the interview

specialized in bilingual assessment, counseling, and consultation. They recognized their potential contributions to serve students and families from culturally and linguistically diverse backgrounds. Notably, they identified their abilities to help families and students with matched racial/ethnic and language backgrounds. For example, one participant noted that "this is really important because in America, in our school, there are a great number of immigrant children. If there is no school psychologist understand that immigrant [children] are undersupport[ed], how are you gonna deal with children issues" (Mary, an Indian international student).

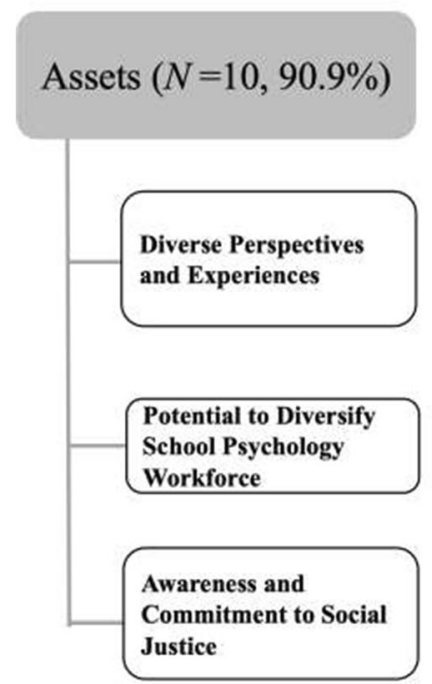

Fig. 1 Diagram of themes and subthemes
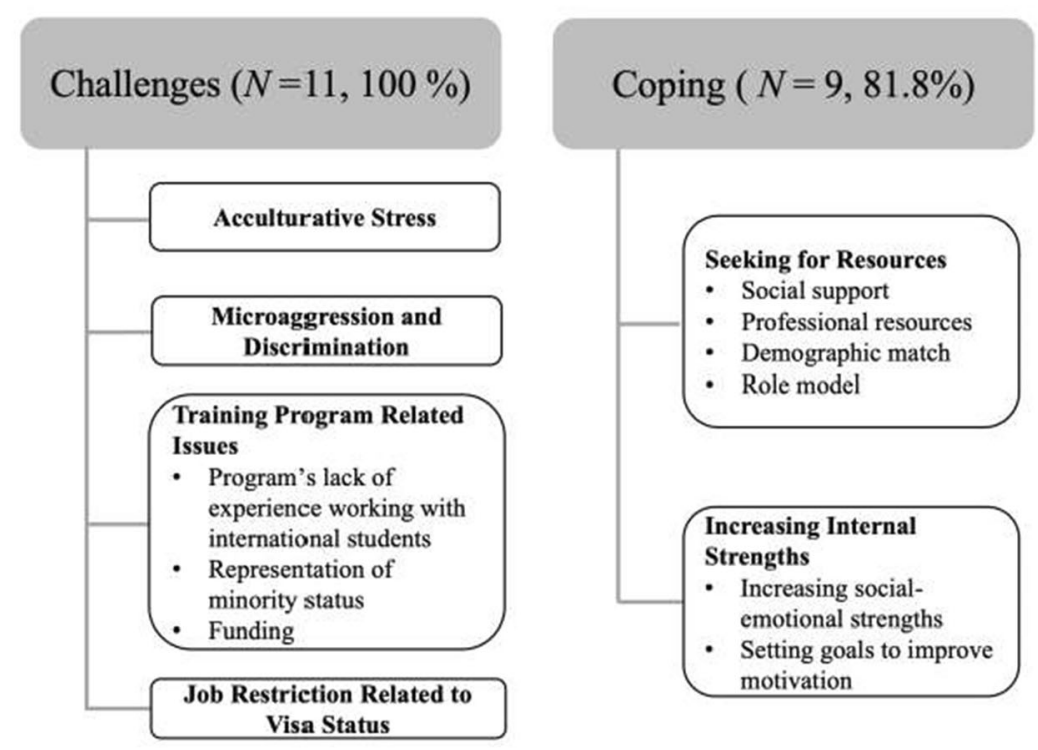


\section{Awareness and Commitment to Social Justice}

About $27.3 \%$ of participants mentioned that their awareness of and commitment to social justice and equity was an essential asset to school psychology. Because of their foreign status in the USA, the drastic shift from being a majority in their home country to being a minority in the USA has made them more aware of social justice and equity issues in the USA (e.g., critical race theory; racial/ethnic disparities; institutional racism). They reported being more attentive and empathetic towards students from marginalized backgrounds (e.g., ethnic minorities). They also expressed a strong commitment to the advocacy and social justice for minority students, as a participant reported that "I tend to pay more attention to these minority students, such as Asian students in [my] class. I would be more attentive to their schooling experiences and wanted to help them at school" (Melissa, a Chinese international student).

\section{Theme 2: Challenges}

Throughout the interviews, the most salient themes recognized by all the participants were the challenges they faced in training. On average, each participant mentioned 15 (SD = 5) challenges during the interview. These challenges are categorized into (a) acculturative stress (e.g., lack of social support, demographic mismatch, language barriers), (b) microaggression and discrimination (e.g., voice not being heard), (c) program-related issues (e.g., lack of representation in the program, program's experience with international students, lack of funding), and (d) visa problem.

\section{Acculturative Stress}

All students (100\%) reported that they were experiencing acculturative stress related to a variety of factors during their training in the programs. The two most commonly identified factors are language barriers and a lack of social support. Regarding language barriers, many participants reported that they were concerned of being perceived by others (faculty members and peers in the program, students, supervisors or staff in the practicum/internship sites) as less competent in English when conducting assessments, delivering evaluation feedback, and writing psychoeducational reports and research manuscripts. Some participants expressed the desire for their programs to offer more multicultural classes and provided more support for report writing. Besides, many participants reported that the lack of social support and feeling of loneliness and isolation contributed to their increased stress. Most of the participants came to the USA alone for their education, and they did not have family members or established social networks when they first arrived in the USA. They often faced high levels of stress when trying to navigate the program and community and build their social network.

Some participants stated that they have experienced cultural shock and heightened self-consciousness, which impeded their learning and engagement in the program/practicum site. Some participants said they noticed that some people (peers, faculty, and school staff) would become impatient with them when they spoke English with accents. Such experiences increased their anxious feelings about whether they have used the wrong vocabulary or made others think they were "socially awkward." Many participants who are non-native English speakers expressed that they constantly ruminate on their vocabulary and behaviors and worry about being negatively evaluated by others at their practicum sites. One participant shared:

Because I myself would perceive or constantly think about whether [my ways of expressing myself] would impact my relationships with the consultees, or even whether it would impact how they perceived me. What I meant is that they might feel what I said lacked authority, or the wording I used might seem socially awkward [to them] (Tom, a Hong Kong international student).

Furthermore, their acculturative stress was also related to the different cultural norms and expectations across US and Asian cultures. Many participants were aware that being assertive is a more desirable personal quality in the USA than in their home countries. Being aware of such a discrepancy, some participants reported the pressure of having to adapt to this new cultural value quickly.

\section{Microaggression and Discrimination}

All participants (100\%) shared that they have been unfairly treated by their peers, faculty members in their training programs, or supervisors at their practicum sites, mainly due to the different cultural backgrounds. Some participants reported explicit degradation, such as being verbally abused by peers (e.g., "you are such an a**, like you work so slow, no one will hire you"). Some reported their experience of being "dismissed" by their professors because they asked "fundamental questions that everybody knew." Some participants commented on getting unfair treatments in the practicum sites and in the program, such as receiving much fewer caseloads than domestic students. Students also received pervasive microaggression, ranging from others having stereotypes on Asian students being submissive and not speaking up. More of concern is that when students shared their concern with faculty, these concerns were dismissed and rationalized by saying, "it was normal for Asians in this program." As a result of their negative experiences in the program, some believed that a big reason why the program accepted them was tokenism over 
their own values as individuals, so that the program could increase the representation of minority and international students.

Participants also expressed a dilemma between experiencing the lack of opportunities to have their voice heard in their programs and their experiences of being punished for speaking up in the programs. Participants also expressed their enthusiasm in school psychology and saw themselves to have a lot to offer to the field. However, they did not feel they had opportunities to speak up. Some students explicitly shared their personal stories about being punished (e.g., being delayed for 1 year) for speaking up their experience of being discriminated in the program. Some students indicated that they learned ways to express their concerns after a few years of struggles.

\section{Training Program-Related Issues}

Program's Lack of Experience Working with International Students All participants (100\%) reported that their training needs as international students were not effectively addressed by their programs. Several participants said that their programs did not have prior experiences working with international students as they were the first Asian international student in the program. Some participants felt they "received inadequate support yet at the same time too much support," which did not sufficiently address the real need of international students. For example, participants shared that their peers, faculty members, or clinical supervisors in the training program often assumed that all students have gone through K-12 schools personally in the USA and thus are familiar with the US school system. Some participants commented on the lack of diversity exposure to diversity in their curriculum as another concern about their programs. One participant described:

We only talk about the white teacher, or educator and [them] interacting with non-majority culture students, you know. But what about the other cultures? You are [educating students] with non-majority background, what are issues for people like us to interact with people like with any other culture? People [from different backgrounds appeared to] have the same issues. I would not know [about] the culture or the knowledge about how to interact with people with different cultural backgrounds like Black students, Hispanic students. I have the same issue, but there is nothing in the literature (Mary, an Indian international student).

Representation of Minority Status A large percentage of participants $(72.7 \%)$ reported that they were usually the only one or one of the very few international students in the entire school psychology program. They often felt that there was a deficient representation of Asian international students in the program and the field of school psychology at large. Some participants also observed a mismatch between their cultural identity and the cultural identity of the majority of students, faculty members, or clinical supervisors in their program. The low minority representation and identity mismatch often made them feel lonely and lack of sense of belonging with the program. Some students also expressed concerns about the lack of racial/ethnic and socioeconomic diversity in their program. They felt that the overrepresentation of White middle-class students in their programs could devalue minority students' voices.

Funding Participants (54.5\%) reported difficulties with securing funding. Some participants noted their ineligibility to apply for state scholarships, due to their international student status. Therefore, their funding opportunities were limited to finding teaching assistant (TA) or certain research assistant (RA) positions in the programs. Moreover, some participants shared their observations about programs selecting domestic students over international students for TA and RA positions, due to the face that English is their second language.

\section{Job Restriction Related to Visa Status}

Another challenge that participants $(45.5 \%)$ described was visa-related issues. With the increasing unfriendly political environment for international students to secure a job in the USA, they found that their legal status could limit their current and future job opportunities. This is mainly due to the fact that international students were restricted from applying for jobs in places where the employer can sponsor their working visas (i.e., H1B), and most school districts do not sponsor H1B visa. Their visa status as an international student also limits the hours and the types of jobs they can work on campus as a student during their training.

\section{Theme 3: Coping}

Nine out of eleven participants $(81.8 \%)$ described the practical coping strategies they used to overcome the challenges. The strategies were summarized as two subthemes, including (1) seeking for resources (i.e., professional and financial resources, demographic and identity match, role models, and social support) and (2) increasing internal strengths (i.e., increasing social-emotional strengths and practicing goals setting).

\section{Seeking for Resources}

Social Support Participants (72.7\%) sought social supports from others to cope with challenges. Many participants sought support from peers, roommates, colleagues, and professors 
who shared similar experiences or being empathetic to their experiences. Some participants also found that it is helpful to seek social support from religious and spiritual organizations. One participant also reported seeking out on-campus student counseling services when addressing their mental health needs.

Professional Resources Participants (63.6\%) described resource-seeking as a learned strategy to meet their needs for finance and professional development. Some participants built their career networks in professional organizations through conferences and social media, such as the National Association of School Psychologists (NASP) and the Association of Chinese Helping Professionals and Psychologists-International (ACHPPI). For language needs, program resources were sought, such as writing workshops and accent reduction support. Strategies to secure financial support included applying for on-campus jobs and potential stipend opportunities in practical sites.

Role Models Participants (45.5\%) also observed that having a role model in the program or the field of school psychology was helpful for their professional growth. Many participants found allies with faculty of color and professors who showed them genuine support. Some participants regarded previous international students, who graduated from school psychology programs, as their role models. They reported being inspired by their accomplishments and meaningful contributions to the field of school psychology. A participant described that "in terms of faculty, we have three faculty members, and one of which is my advisor. She is a Black woman, so basically, we, all the students of color, saw her as a role model. She would share a lot of her experiences with us" (Linda, a Chinese international student).

Demographic Match Participants (18.2\%) felt that they were able to contribute more to the school districts when they intentionally chose to live in the cities where they could serve the population that matched their own racial/ethnic background. For example, some participants' programs had resources and training experiences for students to work with Asian immigrant students in the area. A participant explained that "about the advantage, because we are in [a state in the U.S.], I have visited schools in [a state in the U.S.]. There are many Asian people, and right now, they have many opportunities for bilingual assessments" (Susan, a Chinese international student).

\section{Increasing Internal Strengths}

Fostering Social-Emotional Strengths Participants (63.6\%) learned to effectively apply their social-emotional strengths to manage their emotions/stress, achieve their goals, and establish positive relationships with others. Some participants tried to endorse optimism and perspective taking when experiencing discrimination and microaggression. They also valued being open-minded and actively learning about US mainstream culture to help them adjust. They described the importance of "getting out the comfort zone" to experience and understand the US mainstream cultures. Meanwhile, they explained that it was also important to accept their own culture and felt "welcomed" to ask about their culture openly. One participant talked about trying to educate peers about his culture and prevent microaggression:

[When faced with discriminatory situations,] I said to my professor that "I don't blame people, I blame behaviors. There are just some people who [are not aware of their behaviors], but how about we do something to correct behaviors, [especially because] we are behaviorists, right?" (Sam, a Chinese international student)

Setting Goals to Improve Motivation Participants (18.2\%) reported adopting a goal-setting skill to motivate themselves throughout their graduate training. Many participants expressed passions in the field of school psychology, regardless of the challenges they experienced. They showed a strong sense of resilience to overcome the difficulties, such as "working harder in practicum" and "actively seeking resources in improving their writing skills." Some participants commented that their goal-oriented working attitudes in graduate school paid off, which motivated them in their careers.

\section{Discussion}

To our knowledge, this was the first empirical study focusing on the training experiences of Asian international students in school psychology graduate programs in the USA to explore their perception of the assets, the challenges they have faced in their training program, and the commonly used strategies they used to cope and overcome these challenges. The findings can inform the training of international students in the field of school psychology and how program staff and peers could better develop the strengths of international trainees to contribute to the field and optimize their learning experience in the school psychology program.

\section{Assets of Asian International Students in School Psychology Program}

In our study, about $91 \%$ of the participants recognized that they had brought assets into their school psychology program, including (a) diverse perspectives and experiences, (b) diversified workforce, and (c) awareness and commitment to social 
justice. Participants in the current study described that their experiences of growing, living in, or working in a different country or cultural environment provided them with unique perspectives and experiences. Moreover, some participants mentioned that they became aware of these assets when they shared these experiences with peers that were open-minded and welcoming within the training programs. This finding highlights the importance of a healthy and interactive program context in promoting international school psychologists' assets.

\section{Challenges Faced by Asian International Students in the School Psychology Program}

Acculturative stress was the most salient challenge faced by all participants, not only in their training program but also at their practicum/internship sites. In line with previous studies focusing on the barriers of ethnic minority school psychology students (Clark et al. 2012), many Asian international trainees also expressed high self-consciousness, low sense of belonging, and more difficulty engaging in their learning in the school psychology program. All 11 Asian international students faced microaggressions and discrimination in their program, targeting their languages, accents, and cultural values. Microaggression, along with a low sense of belonging, has been acknowledged as factors causing many African American school psychology trainees to drop out of their program and existing the discipline entirely (Proctor and Truscott 2012). Our results suggest that discrimination and microaggression among Asian international students should be taken seriously instead of being dismissed by program faculty (see "Implications for School Psychology Training").

\section{Effective Coping Strategies Used by Asian International School Psychology Students}

Participants shared coping strategies, including seeking resources and support from programs, social support from others, and practicing their social-emotional strength. For example, they actively sought positions in school districts that match their demographic characteristics to serve a student population that they are most interested in. The strategy of finding a role model was often used to promote their selfesteem and motivations to contribute to the field. Previous studies on counseling and clinical programs have shown that quality mentoring relationships with faculty members positively affect an individual's self-efficacy and satisfaction with the program (Clark et al. 2012; Hollingsworth and Fassinger 2002). Consistent with previous results (Khawaja and Stallman 2011), participants in our study also managed their acculturative stress by seeking support from students from their own country, possibly to find a sense of confirmation and empathy. However, it was also recommended that international students extend their social networking to their domestic peers to cope with acculturative stress (Ujitani and Volet 2008; Lee et al. 2004).

\section{Limitations and Future Directions}

While the present study advanced our understanding of international student's perception of current graduate training in the field of school psychology, there are some limitations. First, eight out of eleven participants were from China. The findings cannot represent all Asian international students. Moreover, eight participants were current students in school psychology programs, while only three have already graduated from their program. Therefore, future studies should recruit a more diverse sample of Asian international students and have a balance in educational status to further understand the different experiences of Asian international students with different academic levels. Additionally, our sample might be biased in a way that interviewees who lacked support from their training programs were more likely to participate and share their experiences. This led to a greater focus on the challenges in our results. Furthermore, guided by an interpretive framework in qualitative data analysis, we acknowledge that the themes that emerged from this study were influenced by the specific context in which they were embedded, including the educational background and subjectivity of the researchers (i.e., current school psychology students or faculties) and the lived experiences of the participants.

\section{Implications for School Psychology Training}

In order to support international students and promote diversity within the field of school psychology, training programs should first recognize the unique cultural, linguistic, and professional assets that international students bring with them and then utilize these assets to benefit domestic school psychology students in the program and the broader student population they will serve. Surprisingly, while all eleven participants were bilingual, only one described her bilingual skills as an asset, whereas many of the participants $(72.7 \%)$ recognized their English language proficiency as a significant barrier. This may reflect that many training programs rely on a deficits-focused approach with international students (e.g., concerned about students' Asian accents during test administration and oral communication), which fails to help students identify and maximize their linguistic and cultural strengths. It is important to note that all these international students were admitted into school psychology graduate programs after they provided sufficient evidence of their English proficiency through test scores (TOFEL, GRE) and interviews. After international students are admitted into the school psychology program, it is harmful to programs to overemphasize students' language to the degree that many students did not view their 
Asian language skills as their asset anymore. It is important for faculty members and site supervisors to use a strength-based supervision approach in which "supervisors and supervisees collaboratively assess and build upon supervisee strengths, including cultural assets" to support international trainees to use their strength to overcome challenges (Newman et al. 2017 , p. 2). It is also crucial for programs to adopt culturally sensitive curricula and integrate multicultural content into their graduate training to increase their students' awareness about their bilingual and multicultural strengths (Newell et al. 2010).

It is very concerning that all international students interviewed in this study experienced microaggression and discrimination by peers, faculty, or site supervisor during their training. Other ethnic minority trainees also share this challenge in school psychology (Proctor and Truscott 2012). While NAPS Practice Model (2010) states that "Understanding and respect for diversity in development and learning, and advocacy for social justice, are foundations for all aspects of service delivery," tolerating discrimination and microaggression in the training program, dismissing students' concerns with discrimination ("it was normal for Asians in this program"), and even punishing students who expressed their interests at the program level are not acceptable. Although we cannot generalize 11 participants' experience to other international students and do not have evidence about the exact prevalence of this concern among international students in school psychology in general, 11 cases of discrimination (or even one case of discrimination) within the program are too many. Although the USA is becoming more diverse, racism has become more prevalent, particularly during COVID-19, such as a surge of racism and hate crime towards Asian Americans and the federal immigration policy limiting international students' access to the USA through eventually rescinded, imposing additional stress on international students. The recent turmoil underscores the ethical and moral responsibility for school psychology training programs to combat inequity and inequality by first addressing discrimination and microaggression towards minority students (Asian international students and other minority students) within the program. To push back against the racism and discrimination in our society, like school psychologists, we have to "address the systemic racism embedded in the organization and our beings to make the authentic and lasting change" (R. Navarro, personal communication, May 30, 2020). As stated in APA Multicultural Guideline 5 (2017), "Psychologists aspire to recognize and understand historical and contemporary experiences with power, privilege, and oppression." Faculty and supervisors have to, first, acknowledge our stereotypes and biases towards Asian international students. Without realizing that faculty and supervisors come from a place of privilege and power, we may be susceptible to the culturally biased judgment of Asian and other minority students.
It is also important to provide faculty members, supervisors, and staff with professional development opportunities, such as implicit bias training to improve their awareness about the stereotypes and implicit biases they have about students from diverse backgrounds and to learn effective strategies to intentionally decrease these biases. Moreover, Zhou et al. (2008) suggested a culture synergy framework introduced as a reciprocal process in which faculty and students learn from each other by proactively trying to understand each other's culture. Supervisors also need to take proactive actions to understand individual differences related to international students' countries of origin, language proficiency, level of acculturation, unique challenges (e.g., visa requirements), and interpersonal styles to provide adequate supervision for international students (Mori et al. 2009), and provide useful career advisor and networking opportunities for students.

Our findings also highlight the importance of having an open and interactive learning environment and call for a change in the current school psychology training programs. To secure international students' sense of community in the program, program faculty and staff should also actively involve international students in program activities and make themselves available to all students. For example, faculty members and staff could help support peer support groups, where space is provided for students to share their voice and concerns and receive feedback from their peers. Moreover, to promote students' confidence and motivation, it is essential to provide faculty members with resources and guidance to build positive mentoring relationships with their advisees and serve as role models. Understanding the specific challenges that international students face in studying in school psychology graduate programs and their coping strategies allows program staff and faculty members to target these barriers better and effectively address the needs of international students.

\section{Conclusion}

This study is the first empirical study investigating the assets, barriers, and coping strategies of Asian international students in the school psychology program in the USA. The findings unveiled ways to assist international and minority trainees and recommend a strength-based training that could cultivate the diverse cultural assets they possessed. The suggestions offered in this work can be adopted by program directors to enhance the current training, bringing local and international impact to the field. The value of embracing and nurturing diversity enables the school psychology community to thrive. Last but not least, international students do not only enrich the training and perspectives of school psychology programs but also extend the impact of the US school psychology programs on global scale. 
Funding The author(s) received no specific funding for this work.

\section{Compliance with Ethical Standards}

This study involved human participants. The study was approved by the Human Subjective Review Board of the researchers' academic institution.

Conflict of Interest The authors declare that they have no conflict of interest.

Informed Consent Informed consent was obtained from all individual participants included in the study.

\section{References}

American Psychological Association. (2017). Multicultural guidelines: an ecological approach to context, identity, and intersectionality. Retrieved from http://www.apa.org/about/policy/multiculturalguidelines.aspx

Braun, V., \& Clarke, V. (2006). Using thematic analysis in psychology. Qualitative Research in Psychology, 3, 77-101. https://doi.org/10. 1191/1478088706qp063oa.

Campbell, J. L., Quincy, C., Osserman, J., \& Pedersen, O. K. (2013). Coding in-depth semi-structured interviews: problems of unitization and intercoder reliability and agreement. Sociological Methods \& Research, 42, 294-320. https://doi.org/10.1177/ 0049124113500475.

Choudaha, R., \& Chang, L. (2012). Trends in international student mobility. World Education News \& Reviews, 25(2).

Clark, C. R., Mercer, S. H., Zeigler-Hill, V., \& Dufrene, B. A. (2012). Barriers to the success of ethnic minority students in school psychology graduate programs. School Psychology Review, 41, 176-192. https://doi.org/10.1080/02796015.2012.12087519.

Fergus, S., \& Zimmerman, M. A. (2005). Adolescent resilience: a framework for understanding healthy development in the face of risk. Annual Review of Public Health, 26, 399-419. https://doi.org/10. 1146/annurev.publhealth.26.021304.144357.

Goldenberg, B. M. (2014). White teachers in urban classrooms: embracing non-white students' cultural capital for better teaching and learning. Urban Education, 49(1), 111-144.

Grayson, J. P. (2008). The experiences and outcomes of domestic and international students at four Canadian universities. Higher Education Research \& Development, 27(3), 215-230.

Hollingsworth, M. A., \& Fassinger, R. E. (2002). The role of faculty mentors in the research training of counseling psychology doctoral students. Journal of Counseling Psychology, 49, 324-330. https:// doi.org/10.1037/0022-0167.49.3.324.

Institute of International Education. (2019). Primary source of funding. Retrieved from https://www.iie.org/Research-and-Insights/OpenDoors/Data/International-Students/Primary-Source-of-Funding

Khawaja, N. G., \& Stallman, H. M. (2011). Understanding the coping strategies of international students: a qualitative approach. Journal of Psychologists and Counsellors in Schools, 21, 203-224. https:// doi.org/10.1375/ajgc.21.2.203.

Lee, K. C. (2013). Training and educating international students in professional psychology: what graduate programs should know. Training and Education in Professional Psychology, 7, 61-69. https://doi.org/10.1037/a0031186.

Lee, J. S., Koeske, G. F., \& Sales, E. (2004). Social support buffering of acculturative stress: a study of mental health symptoms among Korean international students. International Journal of Intercultural Relations, 28, 399-414. https://doi.org/10.1016/j. ijintrel.2004.08.005.
Mittal, M., \& Wieling, E. (2006). Training experiences of international doctoral students in marriage and family therapy. Journal of Marital and Family Therapy, 32, 369-383. https://doi.org/10.1111/j.17520606.2006.tb01613.x.

Mori, Y., Inman, A. G., \& Caskie, G. I. (2009). Supervising international students: relationship between acculturation, supervisor multicultural competence, cultural discussions, and supervision satisfaction. Training and Education in Professional Psychology, 3, 10-18. https://doi.org/10.1037/a0013072.

National Association of School Psychologists (2010). NASP practice model. Bethesda, MD. Retrieved from https://www.nasponline. org/standards-and-certification/nasp-practice-model/nasp-practicemodel-implementation-guide/section-i-nasp-practice-modeloverview/nasp-practice-model-10-domains

National Association of School Psychologists. (2017). Shortages in school psychology: challenges to meeting the growing needs of U.S. students and schools. Bethesda, MD: Author.

Newell, M. L., Nastasi, B. K., Hatzichristou, C., Jones, J. M., Schanding Jr., G. T., \& Yetter, G. (2010). Evidence on multicultural training in school psychology: recommendations for future directions. School Psychology Quarterly, 25, 249-278. https://doi.org/10.1037/ a0021542.

Newman, D. S., Guiney, M., \& Silva, A. E. (2017). Strengths-based supervision in school psychology: accentuate the positive, eliminate the negative. NASP Communiqué, 46(1), 1.

Park, H., Lee, M. J., Choi, G. Y., \& Zepernick, J. S. (2017). Challenges and coping strategies of East Asian graduate students in the United States. International Social Work, 60, 733-749. https://doi.org/10. $1177 / 0020872816655864$.

Park-Saltzman, J., Wada, K., \& Mogami, T. (2012). Culturally sensitive mentoring for Asian international students in counseling psychology. The Counseling Psychologist, 40, 895-915. https://doi.org/10. 1177/0011000011429831.

Proctor, S. L., \& Truscott, S. D. (2012). Reasons for African American student attrition from school psychology programs. Journal of School Psychology, 50, 655-679. https://doi.org/10.1016/j.jsp. 2012.06.002.

Ra, Y. A., \& Trusty, J. (2015). Coping strategies for managing acculturative stress among Asian international students. International Journal for the Advancement of Counselling, 37, 319-329. https:// doi.org/10.1007/s10447-015-9246-3.

Reid, L. M., \& Dixon, A. L. (2012). The counseling supervision needs of international students in U.S. institutions of higher education: a culturally-sensitive supervision model for counselor educators. Journal for International Counselor Education, 4, 29-41.

Trice, A. G. (2003). Faculty perceptions of graduate international students: the benefits and challenges. Journal of Studies in International Education, 7, 379-403. https://doi.org/10.1177/ 1028315303257120 .

Ujitani, E., \& Volet, S. (2008). Socio-emotional challenges in international education: insight into reciprocal understanding and intercultural relational development. Journal of Research in International Education, 7, 279-303. https://doi.org/10.1177/ 1475240908099975.

Walcott, C. M., Charvat, J. L., McNamara, K. M., \& Hyson, D. M. (2016). School psychology at a glance: 2015 member survey results. Special session presented at the annual convention of the National Association of School Psychologists, New Orleans, LA.

Wang, J. (2009). A study of resiliency characteristics in the adjustment of international graduate students at American universities. Journal of Studies in International Education, 13, 22-45. https://doi.org/10. 1177/1028315307308139.

Wedding, D., McCartney, J. L., \& Currey, D. E. (2009). Lessons relevant to psychologists who serve as mentors for international students. Professional Psychology: Research and Practice, 40, 189-193. https://doi.org/10.1037/a0012249. 
Zhou, Y., Jindal-Snape, D., Topping, K., \& Todman, J. (2008). Theoretical models of culture shock and adaptation in international students in higher education. Studies in Higher Education, 33, 6375. https://doi.org/10.1080/03075070701794833.

Publisher's Note Springer Nature remains neutral with regard to jurisdictional claims in published maps and institutional affiliations.

Chunyan Yang, PhD is an assistant professor of school psychology in the Graduate School of Education at the University of California, Berkeley. Her research interests focus on understanding how school members (e.g., students, teachers, parents) interact with their living contexts (e.g., school, family, community, culture) to find their resilience in face of a variety of risk factors in school settings, such as bullying, teacher-targeted violence, and mental health challenges.

Chun Chen, MEd is a doctoral candidate in the Department of Counseling, Clinical, and School Psychology at the University of California, Santa Barbara. Her research interests focus on school climate, bullying victimization, cross-cultural differences, and family-school engagement.
Meiki Chan is a doctoral student in the Department of Counseling, Clinical, and School Psychology at the University of California, Santa Barbara. Her research interests focus on social-emotional health, crosscultural comparisons, mental health, and bullying victimization.

Cixin Wang, PhD is an associate professor of School Psychology in the Department of Counseling, Higher Education, and Special Education at the University of Maryland, College Park. Her research focuses on bullying prevention, mental health promotion, school climate, and parenting, especially for youth from culturally and linguistically diverse backgrounds.

Hua Luo, MEd is a doctoral student in the school psychology program in the Graduate School of Education at the University of California, Berkeley. Her research interests focus on the impacts of various socialecologic factors (e.g., family, school, peers, community) on adolescent's ethnic-racial identity development and socio-emotional development.

Xueqin Lin, MEd is a doctoral student in the school psychology program in the Graduate School of Education at the University of California, Berkeley. Her primary research interests focus on adolescents' risk and resilience and their impacts on adolescents' academic and mental health outcomes. She is also interested in cross-cultural comparisons, and ethnic identity development, and mental health of immigrant adolescents. 